\title{
Energy Aware Virtual Machine Migration Techniques for Cloud Environment
}

\author{
Kamali Gupta \\ Department of CSE \\ MMU, Sadopur
}

\author{
Vijay Katiyar, PhD \\ Department of CSE \\ MMU, Mullana
}

\begin{abstract}
Cloud Computing offers indispensable infrastructure for storage and computing facilities for development of diversified services. The large utilization of resources leads to increased energy consumption that has imposed a limit on performance growth. Owing to high operational costs and carbon dioxide footprints, an efficient energy management technique needs to be developed and deployed that reduces overall energy consumption of a cloud environment while maximizing the resource utilization. In the first phase of this research, some virtual machine migration techniques were explored. In the second phase, a virtual machine migration technique has been implemented which aims at reducing energy consumption in cloud datacentres.
\end{abstract}

\section{General Terms}

Virtual Machine Migration, Bin Packing Algorithms.

\section{Keywords}

Cloud Computing, Virtualization, Energy Management.

\section{INTRODUCTION}

Cloud Computing [1] is a provider of dynamic and diversified services over internet by utilizing virtualized and scalable resources. It is known as service oriented paradigm as it provides "everything as a service". A cloud can be termed as an execution environment of resources which provides metered services at multiple levels to multiple stakeholders in a very efficient and elastic manner. It allows for execution of applications and services in a much managed way. The term "managed" ensures reliability in its operations according to already stated quality parameters. Elasticity depicts dynamism and scalability in resource utilization according to current requirements.

The typical services of cloud that can be shared over internet include platform, software's and infrastructure [2]. The term Cloud Computing elaborates the platform and the type of application. As a platform, cloud configures, reconfigures and supplies servers that can be physical machines or virtual machines. As a computing facility, it includes applications that are accessible over internet through supporting large data centres. Data centres are composed of powerful servers that aims to host web application and services [3]. Cloud systems [4] can be classified into three broad categories, namely, private clouds, public clouds and hybrid clouds.

The provider of cloud services multiplexes heterogeneous demands of users for computing resources such as bandwidth, storage, CPU etc. through virtualization technique. The technique of virtualization [5] aims to maximize the utilization of available resources such as network, storage, processor etc. It aims to reduce cost of IT operations by combining a number of idle resources together to create shared pools. It can be accomplished by creating virtual machines that operates simultaneously. Virtualization technique includes the process of VM creation, placement and migration. With the help of such technology, a single data centre or high power server can be sliced to act as multiple machines. The number of virtual machine, a system may be divided, depends upon the hardware configuration of system. Therefore, virtualization is an efficient technique that increases resource utilization and thus helps to conserve energy.

The cloud data centres are capable of housing a large number of IT equipments which consumes enormous amount of energy for their services. The increase in amount of energy consumption has become a major concern for cloud data centres as it leads to large emission of carbon dioxide, higher operational cost and thus shorter life of hardware equipments [6]. The virtualization technology helps in improving power efficiency of the data centres by consolidating the workload of several physical machines onto a single machine by creating multiple VMs. As a result, many physical machines gets turned off because of shifting of load [7][8]. Thus, virtualization technique refers to abstraction of computer resources (such as CPU, storage, network, memory, application stack and database) from the applications and the end users consuming the service [9][10].Energy Management Techniques in cloud are implemented both at hardware and software level as shown in Fig.1. At hardware level, the hardware devices are monitored for reducing the overall consumption of energy and at software level, the virtualization technique helps in reduction in energy consumption.

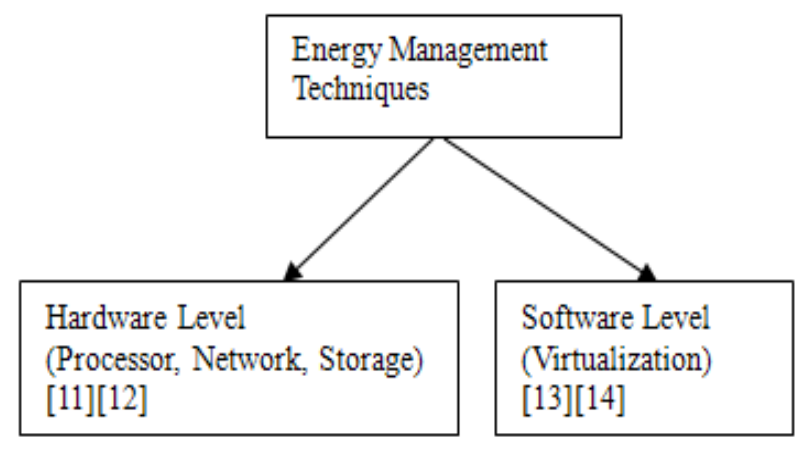

Figure 1 Energy Management Techniques

\section{LITERATURE SURVEY}

The existing solutions for energy efficient resource allocation cannot be implemented for Green computing as they only focus on minimizing the energy consumption in cloud environments or on minimizing the operational cost. Such solutions do not take into account the dynamic and heterogeneous needs of cloud users and their applications [15]. The technique of power-aware scheduling has been addressed that reduces CPU energy consumption through dynamic voltage scaling in hard real-time systems. Some existing intertask voltage scheduling schemes have been explored and on the basis of study a new technique is proposed that outperforms existing techniques. 
Influential work of [16] illustrates challenges, architectural elements and vision for energy-efficient management of components of cloud. Dynamic resource provisioning has been considered as major force driving cloud environments. Stress has been laid on establishing synergy between several infrastructural resources of a data centre. The research work proposes energy-efficient policies for allocation of resources, architectural principles for energy-efficient cloud management, a novel algorithm (software) for energyefficient cloud management.

The survey done in [17] presents various resource utilization techniques that has been used to make data centres more energy efficient. The techniques explored in their research work, utilized the concept of virtualization to reduce the energy consumption and a comparison has been made with other existing computing architectures.

The research work carried out in [18] elaborated virtual machines as isolated boxes that shares and offers resources as when needed and serves the clients in the way as the real servers do. These machines are connected to each other via same or a different network. The concept of Hypervisor is discussed and has been elaborated as a mini operating system capable of running several virtual machines by supervising and controlling them through establishment of communication and resource sharing. The advantage of virtualization as discussed in the research work is "high availability". Virtual machines are formulations of software's which can be copied to other locations without difficulties, if any trouble occurs to the physical servers or its related devices. A brief description of network and storage virtualization has been depicted. In the last section, the use of virtualization in different layers of cloud services model is presented.

In [19], survey of virtualization in cloud computing has been done. The research work explains possible threats for cloud service users and cloud service providers. Additionally, several attacks on cloud computing has also been discussed. Further, some potential solutions for handling these attacks have been proposed.

Techniques have been elaborated in [20] with which virtualization enhances security in a cloud environment. It has been discussed that by protecting both the integrity of cloud infrastructure components and guest virtual machines, security can be increased. An Advanced Cloud Protection System (ACPS) has been proposed that can be deployed on various cloud solutions for guaranteeing increased security in cloud paradigm. ACPS is capable of effectively monitoring the infrastructure components and integrity of guests while remaining completely transparent to cloud users and virtual machines. ACPS has been deployed and tailored onto various cloud implementations. ACPS has been implemented on current open source solutions. The proposed approach has been found to be effective and protects machines from several attacks.

The survey work presented in [21] includes mechanisms with which efficiency in cloud data centres of task completion can be enhanced. The study explores various cloud computing techniques such as virtualization, energy management and resource allocation. The primarily goal of the research work conducted was to walk around the techniques that leads to reduced energy consumption in data centres.

With the implementation of increased computing in consumer, scientific and business domains, profound concerns have risen up relating to tremendous energy consumption and associated costs. In [22], solutions for
Green Cloud Environment have been presented that aims to minimize its environmental impact. It can be accomplished by taking into consideration static and dynamic portions of the cloud components. The proposed methodology presents a generic model to capture cloud computing data centres. Some energy consumption patterns have been investigated and it has been concluded that by applying appropriate optimization policies, $20 \%$ of energy consumption can be saved in cloud environment.

The research work presented in [23] explores all areas that lead to increased energy consumption in a typical cloud environment. Additionally, some methodologies have been addressed that can decrease power utilization without compromising overall performance and Quality of Service. Power Usage Effectiveness (PUE) and Data Centre Infrastructure Efficiency (DCIE) are two measures that have been used to calculate energy consumption in a data centre. The next section presents a brief survey of energy efficient resource scheduling. It has been concluded that few components of cloud architecture are responsible for increased amount of power dissipation such as host machines, IT equipment etc.

\section{VIRTUAL MACHINE MIGRATION TECHNIQUES}

Given a number of virtual machines and physical servers, a feasible placement of available VM's onto the physical machines is to be derived that minimizes the total energy spent by the active physical machines. Existing Bin Packing virtual machine migration techniques have been explored and implemented in this section. Further, a new virtual machine migration technique has been developed and implemented that reduces the energy consumption in a cloud environment.

\subsection{Existing Algorithms [24]}

\subsubsection{Low Perturbation Bin Packing Algorithm $(L P B P)$}

LPBP technique for VM Migration focuses on keeping the number of migrations less by slowing down the transition between the existing and a new VM-server assignment scheme. The list of servers is maintained in descending order of their computing capacities. The algorithm initiates by calculating the power consumption by each server. Then, it computes the total energy spent in the data centre. Finally, it migrates the virtual machines of the most energy consuming server to the least energy consuming server, provided the total computing capacity consumed by virtual machines ( including the newly migrated VM's) of the server (on which migration is to be performed) should not exceed the server's total computing capacity. Else, no migration will be performed and the considered server is to be removed from the server list.

$\mathrm{S}_{\mathrm{x}}=$ Least energy consuming server

$\mathrm{S}_{\mathrm{y}}=$ Most energy consuming server

$\mathrm{CPU}_{\mathrm{i}}=$ Computing capacity of server $\mathrm{x}$.

$\mathrm{U}_{\mathrm{i}}(\mathrm{F}, \mathrm{t})=$ Utilization of $\mathrm{S}_{\mathrm{i}}$ as a function of placement.

$P_{i}(F, t)=$ Power consumption of a server $S_{i}$.

$\mathrm{P}_{\max }=$ Maximum power consumed by a server.

$\operatorname{Re} q_{-} C P U_{x}^{i}=$ Computing requirement of a $\mathrm{VM}$ on $\mathrm{k}$ server.

$\mathrm{N}=$ Number of servers. 
$\mathrm{T}_{-} \mathrm{CPU}_{\mathrm{i}}=$ Total Computing requirement of all VMs on Server $\mathrm{S}_{\mathrm{i}}$.

\section{Algorithm:}

1. Initialize capacity requirements for $\mathrm{Req}_{-} \mathrm{CPU}_{\mathrm{i}}, \mathrm{CPU}_{\mathrm{i}}$ and $P_{\max }$ for each server $S_{i}$.

2. Randomly assign VM onto server such that each server is allotted atleast one VM.

3. Find Energy Consumption of each server $S_{i}$ as $P_{i}(F, t)$

$$
\mathbf{P}_{\mathbf{i}}(\mathbf{F}, \mathbf{t})=\mathbf{0 . 7} p_{\text {max }}^{i}+\mathbf{0 . 3} p_{\text {max }}^{i} * \mathbf{U}_{\mathbf{i}}(\mathbf{F}, \mathbf{t})
$$

Where

$$
\mathbf{U}_{\mathbf{i}}(\mathbf{F}, \mathbf{t})=\sum_{K=1}^{V} F_{\mathbf{i k}} *\left(\operatorname{Req}_{-} \mathbf{C P U}_{\mathbf{i}}(\mathbf{t}) / \mathbf{C P U}_{\mathbf{i}}\right)
$$

4. Arrange the servers in descending order of their energy consumption.

5. Compute the total energy consumption of the system.

6. Compute T_CPU $\mathbf{U}_{\mathbf{y}}=\sum \operatorname{Re} q_{-} C P U_{x}^{i}$

7. If $T_{-} \mathbf{C P U}_{\mathrm{v}}+\mathrm{T}_{-} \mathbf{C P U}_{\mathrm{x}}<=\mathrm{CPU}_{\mathrm{x}}$ then

a. Migrate the VMs of $S_{v}$ to the $S_{x}$.

b. Switch off $S_{y}$ and delete its entry from server list.

8. Compute the total energy consumption of the system.

\subsubsection{Best Fit Decreasing (BFD)}

$\mathrm{BFD}$ is a bin-packing algorithm whose main goal is to reduce the total energy spent in virtualized cloud environment. Initially, the algorithm considers all the servers to be unused and unassigned. Then, on the basis of computing capacity of servers, the VM's are assigned to them. The assignment starts with the server having minimum computing capacity. The VM's list is maintained in descending order of their computing capacity. The maximum capacity demanding VM is considered first and gets placed on minimum computing capacity server. This process continues until the entire VM's are allocated to the servers or the computing capacity of the considered and placed VM's on a server exceeds the server's computing capacity. Once, the placement is done, the total energy spent in the cloud environment is calculated according to the method followed in LPBP.

\section{Algorithm:}

1. Initialize capacity requirements for Req_CPU,$C_{i} U_{i}$ and $P_{\max }$ for each server $S_{i}$.

2. Arrange the VM's in descending order of their computing capacities in VM list.

3. Arrange the servers in ascending order of their computing capacities in Server list.

4. Repeat step 5 until all VM's are allocated

5. For each server $S_{i}$, assign the VM from top of the list to $S_{i}$ such that, $T_{-} C_{P U}<=C P U_{i}$

Where

$$
\mathbf{T}_{-} \mathbf{C P U} \mathbf{U}_{\mathrm{i}}=\sum \operatorname{Re} q_{-} C P U_{x}^{i}
$$

6. Switch off all the servers which do not contain any VM and delete the entry from Server list.

7. Find Energy Consumption of each server $S_{i}$ as $P_{i}(F, t)$

$$
\mathbf{P}_{\mathbf{i}}(\mathbf{F}, \mathbf{t})=\mathbf{0 . 7} p_{\text {max }}^{i}+\mathbf{0 . 3} p_{\text {max }}^{i} * \mathbf{U}_{\mathbf{i}}(\mathbf{F}, \mathbf{t})
$$

Where

$$
\mathbf{U}_{\mathbf{i}}(\mathbf{F}, \mathbf{t})=\sum_{K=1}^{V} F_{\mathbf{i k}} *\left(\operatorname{Req}_{-} \mathbf{C P U} \mathbf{U}_{\mathbf{i}}(\mathbf{t}) / \mathbf{C P U}_{\mathbf{i}}\right)
$$

8. Compute the total energy consumption of the system.

\subsubsection{Power and Computing capacity-Aware Best} Fit Decreasing (PCA-BFD)

PCA-BFD is a modified algorithm which works in the same manner as BCD does. The only difference lies in the consideration of servers for initial placement of VM's. Here, the servers are considered for allocation on the basis of $\mathrm{P}_{\max } /$ $\mathrm{CPU}_{\mathrm{i}}$ ratio. Then, the computed value for servers is arranged in increasing order. The server having the minimum $\mathrm{P}_{\max } /$ $\mathrm{CPU}_{\mathrm{i}}$ value is allotted the maximum capacity VM's first and then the same process follows up as it was discussed in BFD.

\section{Algorithm:}

1. Initialize capacity requirements for $R e q \_C P U_{i}, C P U_{i}$ and $P_{\max }$ for each server $S_{i}$.

2. Calculate $P_{\max } / C P U_{i}$ for each server $S_{i}$.

3. Arrange the servers in ascending order of $\mathbf{P}_{\max } / \mathbf{C P U}_{i}$ value in the server list.

4. Arrange the VM's in descending order of their computing capacity in VM list.

5. For each server $S_{i}$, assign the VM from top of the list to $S_{i}$ such that, $T_{-} C_{C P U}<=C P U_{i}$

Where

$$
\mathbf{T}_{-} \mathbf{C P U}_{\mathrm{i}}=\sum \operatorname{Re} q_{-} C P U_{x}^{i}
$$

6. Switch off all the servers which do not contain any VM and delete the entry from Server list.

7. Find Energy Consumption of each server $S_{i}$ as $P_{i}(F, t)$

Where

$$
\mathbf{P}_{\mathbf{i}}(\mathbf{F}, \mathbf{t})=0.7 p_{\text {max }}^{i}+\mathbf{0 . 3} p_{\text {max }}^{i} * \mathbf{U}_{\mathbf{i}}(\mathbf{F}, \mathbf{t})
$$

$$
\mathbf{U}_{\mathbf{i}}(\mathbf{F}, \mathbf{t})=\sum_{K=1}^{V} F_{\mathbf{i k}} *\left(\operatorname{Req}_{-} \mathbf{C P U}_{\mathbf{i}}(\mathbf{t}) / \mathbf{C P U}_{\mathbf{i}}\right)
$$

8. Compute the total energy consumption of the system.

\subsection{Developed Algorithm}

\subsubsection{Proposed Algorithm}

Proposed Algorithm is a bin-packing algorithm whose main goal is to reduce the total energy spent in virtualized cloud environment. Initially, the algorithm considers all the servers to be unused and unassigned. Then, on the basis of power consumption of servers, the VM's are assigned to them. The assignment starts with the server whose maximum power consumption value is least. The VM's list is maintained in ascending order of their computing capacity. The minimum capacity demanding VM is considered first and gets placed on server having its $\mathrm{P}_{\max }$ as the least value among the other servers. Further, if two or more servers have same $\mathrm{P}_{\max }$ value, then allotment is done on the basis of their computing capacities of servers. This process continues until the entire VM's are allocated to the servers or the computing capacity of the considered and placed VM's on a server exceeds the server's computing capacity. Once, the placement is done, the total energy spent in the cloud environment is calculated according to the method followed in BFD.

\section{Algorithm:}

1. Initialize capacity requirements for Req_CPU,$C_{i} \mathbf{P U}_{\mathbf{i}}$ and $P_{\max }$ for each server $S_{i}$.

2. Arrange the VM's in increasing order of their computing capacity.

3. Arrange the servers in increasing order of their maximum power requirements.

4. Servers having same $P_{\max }$ value are arranged in descending order of their computing capacities.

5. Repeat step 6 until all VM's are allocated.

6. For each server $S_{i}$, assign the VM from top of the list Where 


$$
\mathbf{T}_{-} \mathbf{C P U}_{\mathrm{i}}=\sum \operatorname{Re} q_{-} C P U_{x}^{i}
$$

7. Switch off all the servers which do not contain any VM and delete the entry from Server list.

8. Find Energy Consumption of each server $S_{i}$ as $P_{i}(F, t)$

$$
\mathbf{P}_{\mathbf{i}}(\mathbf{F}, \mathbf{t})=0.7 p_{\max }^{i}+\mathbf{0 . 3} p_{\text {max }}^{i} * \mathbf{U}_{\mathbf{i}}(\mathbf{F}, \mathbf{t})
$$

Where

$$
\mathbf{U}_{\mathbf{i}}(\mathbf{F}, \mathbf{t})=\sum_{K=1}^{V} F_{i \mathbf{i k}} *\left(\operatorname{Req}_{-} \operatorname{CPU}_{\mathbf{i}}(\mathbf{t}) / \mathbf{C P U}_{\mathbf{i}}\right)
$$

9. Compute the total energy consumption of the system.

\section{PERFORMANCE ANALYSIS}

A comparison among the four algorithms in terms of the total energy spent per unit time is presented. Computing capacities for $m$ servers and $n$ virtual machines are randomly generated to find a suitable placement such that the overall energy consumption can be minimized. The maximum computing capacity of servers, maximum power utilization of the servers and maximum computing capacity of virtual machines has been kept as static. On the basis of generated values, maximum energy consumption is calculated according to the implemented VM techniques. Taking the value of CPU_Max=5000, Pmax_Max=1500 and VM_Max $=450$, energy consumption in proposed algorithm in comparison with other existing algorithms is presented in Table 1, 3\& 5 and Figure 2, $3 \& 4$.

Table 1 Simulation results with less Servers and VMs

\begin{tabular}{|l|c|c|c|}
\hline \multicolumn{4}{|c|}{ CPU_Max= 5000, Pmax_Max=1500, VM_Max=450 } \\
\hline \multicolumn{4}{|c|}{ No. of Servers = 10, No. Of VMs= 30 } \\
\hline LPBP & BFD & PCA-BFD & EBFD' \\
\hline 1959.61 & 2714.06 & 1733.63 & 1365.35 \\
\hline
\end{tabular}

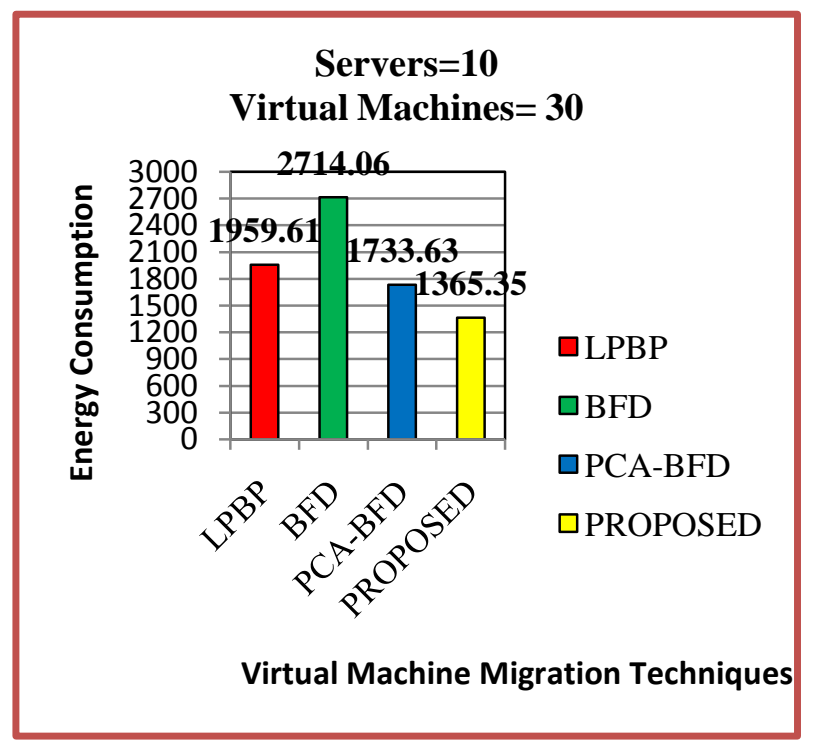

Figure 2 Energy Consumption with lesser machines

Figure 2 represents energy consumption by VM migration techniques when the computing capacities and the power requirements have been kept as static. 10 servers and 30 virtual machines are taken to compute the total energy consumption. Energy reduction (in percentage) in proposed algorithm in comparison to existing algorithms LPBP, BFD, PCA-BFD is presented in Table 2.
Table 2 Energy Reduction by proposed technique in comparison to existing techniques with less Servers \& VMs.

\begin{tabular}{|l|l|}
\hline VM Technique & Energy Reduction(In Percentage) \\
\hline LPBP & 30.32 \\
\hline BFD & 49.69 \\
\hline PCA-BFD & 21.24 \\
\hline
\end{tabular}

Table 3 Simulation results with average Servers and VMs

\begin{tabular}{|l|c|c|c|}
\hline \multicolumn{4}{|c|}{ CPU_Max= 5000, Pmax_Max=1500, VM_Max=450 } \\
\hline \multicolumn{4}{|c|}{ No. of Servers = 10, No. Of VMs= 30 } \\
\hline LPBP & BFD & PCA-BFD & EBFD' \\
\hline 4411.56 & 5912.83 & 5326.81 & 3359.18 \\
\hline
\end{tabular}

Figure 3 represents energy consumption with 25 servers and 60 vms. Energy reduction (in percentage) in proposed algorithm in comparison to existing algorithms is presented in Table 4.

Table 4 Energy Reduction by proposed technique in comparison to existing techniques with average Servers \& VMs.

\begin{tabular}{|l|l|}
\hline VM Technique & Energy Reduction(In Percentage) \\
\hline LPBP & 23.85 \\
\hline BFD & 43.18 \\
\hline PCA-BFD & 36.93 \\
\hline
\end{tabular}

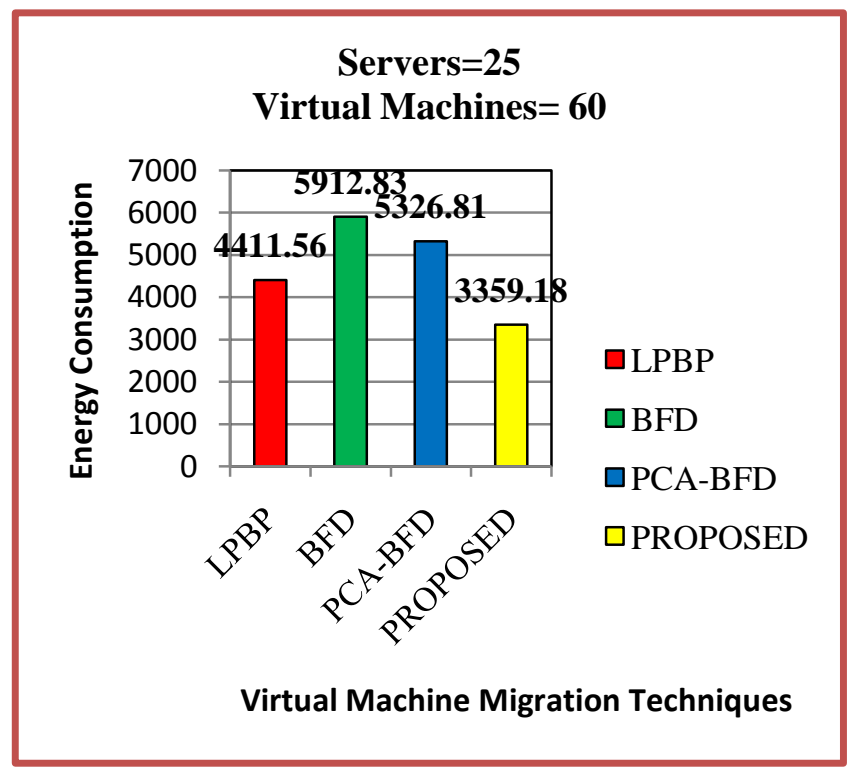

Figure 3 Energy Consumption with average machines

Figure 4 represents energy consumption with 48 servers and 88 virtual machines. 


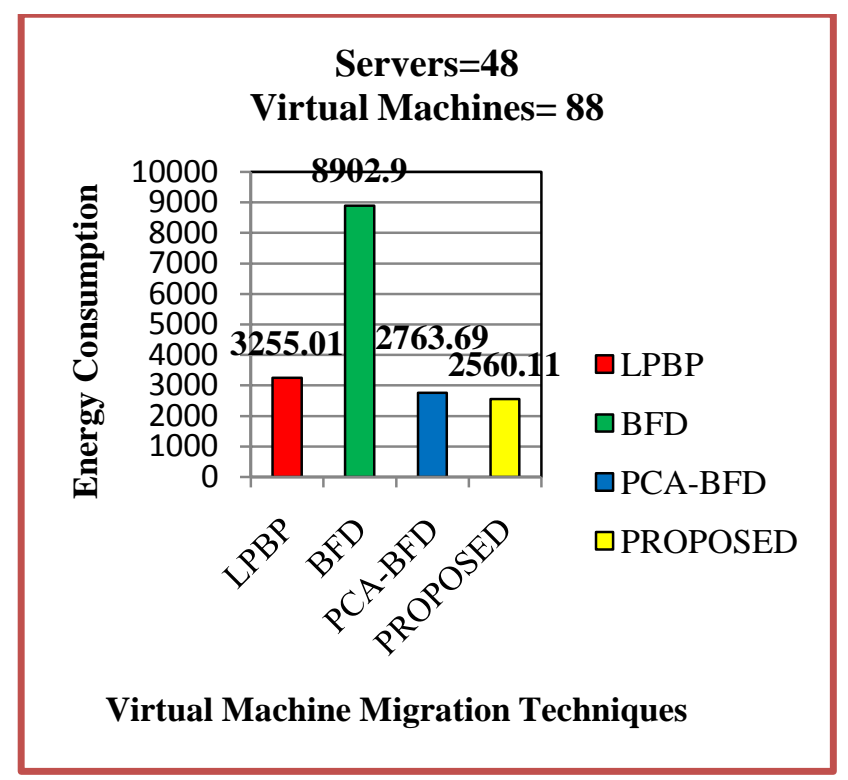

Figure 4 Energy Consumption with more machines

Table 5 Simulation results with average Servers and VMs

\begin{tabular}{|l|c|c|c|}
\hline \multicolumn{4}{|c|}{ CPU_Max= 5000, Pmax_Max=1500, VM_Max=450 } \\
\hline \multicolumn{4}{|c|}{ No. of Servers = 10, No. Of VMs= 30 } \\
\hline LPBP & BFD & PCA-BFD & EBFD' \\
\hline 3255.01 & 8902.9 & 2763.69 & 2560.11 \\
\hline
\end{tabular}

Energy reduction (in percentage) in proposed algorithm in comparison to existing algorithms LPBP, BFD, PCA-BFD presented in Table 6 .

Table 6 Energy Reduction by proposed technique in comparison to existing techniques with average Servers \& VMs.

\begin{tabular}{|l|l|}
\hline VM Technique & Energy Reduction(In Percentage) \\
\hline LPBP & 21.34 \\
\hline BFD & 71.24 \\
\hline PCA-BFD & 7.36 \\
\hline
\end{tabular}

\section{CONCLUSION}

In the research work, focus has been directed towards deriving a virtual machine migration technique that reduces the overall energy consumption in cloud data centres. As depicted in Figure 1, energy management techniques can be implemented at both hardware and software levels. The research work was oriented towards contributing in reducing energy consumption at software level. To attain this, some existing virtual machine migration techniques were explored and on the basis of study, a new technique was proposed and implemented. Simulation results show that the proposed technique was efficacious in reducing energy consumption in a number of VM-server placements for effective VM migrations with less, average and good number of servers and virtual machines.

Simulating the proposed algorithm with higher number of servers and virtual machines on a virtualized data centre to reduce the energy consumption considerably constitutes the future research work.

\section{ACKNOWLEDGEMENTS}

We thank the experts from Maharishi Markandeshwar University who are endowed with insight and expertise which extensively contributed in research findings and development of this paper.

\section{REFERENCES}

[1] S.Pinal, "A Survey of Various Scheduling Algorithm in Cloud Computing Environment", International Journal of Research in Engineering and Technology, ISSN 2319-1163, Volume 2, Issue (2), 2013, 131-135.

[2] S.Marston, Z. Li, S. Bandyopadhyay, J. Zhang and A. Ghalasi, "Cloud Compuating- The business perspective", Decision Support Systems, Volume 51, Issue(1), 176-189, 2011.

[3] The NIST Definition of Cloud Computing, http://csrc.nist.gov/publications/nistpubs/800145/SP800-145.pdf.

[4] Z.Zhang, Richard T.B. Ma, J.Ding and Y.Yang, “ ABACUS: An Auction-Based Approach to Cloud Service Differentaition", IEEE, International Conference on Cloud Engineering, 2013, 292-301.

[5] M.Durairaj and P.Kannan, "A Study on Virtualization Techniques and Challenges in Cloud Computing", International Journal Of Scientific \& Technology Research, Volume 3, Issue (11), 2014, 147-151.

[6] T. Veni and S. Mary, "Dynamic Energy Management in Cloud Data Centers: A Survey", International Journal on Cloud Computing: Services and Architecture, Volume 3, Issue (4), 2013, 13-26.

[7] Pragya and G..Manjeet, “A Review on Energy Efficient Techniques in Green Cloud Computing”, International Journal of Advanced Research in Computer Science and Software Engineering, Volume 5, Issue (3), 2015, 550554.

[8] C. Gong, J. Liu, Q. Zhang, H. Chen, and Z. Gong, "The characteristics of cloud computing", IEEE International Conference on Parallel Processing Workshops, 2010, 275-279.

[9] A. Younge, R. Henschel, T. Brown, G. Laszewski, J. Qiu and G. Fox, "Analysis of virtualization technologies for high performance computing environments", IEEE, International Conference on Cloud Computing, 2011, 9-16.

[10] R.Mietzner, T. Unger, R. Titze and F. Leymann, "Combining different multi-tenancy patterns in serviceoriented applications", IEEE International Conference on Enterprise Distributed Object Computing, 2009, 131 140.

[11] A.Carpen-Amarie, A.C. Orgerie and C.Morin, "Experimental Study on the Energy Consumption in IaaS Cloud Environments", IEEE/ ACM $6^{\text {th }}$ International Conference on Utility and Cloud Computing, 2013, 4249.

[12] S.Jing, S.Ali, K.She and Y.Zhong, "State-of-the-art research study for green cloud computing", Journal of Supercomputing, Vol. 65(1), 2013, 445-468.

[13] L.Deboosere, B.Vankeisbilck, P.Simeons, F.Turck, B.Dhoedt and P.Demeester, "Efficient resource management for virtual desktop cloud computing', Journal of Supercomputing, Volume 62, Issue (2), 2012, 741-767. 
[14] F.Fakhar, B.Javed, R.U.Rasool, O.Malik and K.Zulfiqar, "Software level green computing for large scale systems", Journal of Cloud Computing:Advances, Systems and Applications, 2012.

[15] H. Aydin,R. G. Melhem, D. Mossé, and P. Mejía Alvarez, "Power-Aware Scheduling for Periodic Real Time Task", IEEE, Transactions on Computers, Volume 53, Issue (5), 2004, 584-600.

[16] R.Buyya, A.Beloglazov and J.Abawajy, “ EnergyEfficient Management of Data Centre Resources for Cloud Computing: A Vision, Architectural Elements, and Open Challenges", Cloud Computing and Distributed Systems Laboratory, Australia, 2010, 1-12.

[17] D. Akshat and P. Sanchita, "A Survey of Energy Efficient Data Centres in a Cloud Computing Environment", International Journal of Advanced Research in Computer and Communication Engineering, Volume 2, Issue(10), 2013, 4033-4040.

[18] K.Kamyab, "Role of Virtualization in Cloud Computing", International Journal of Advance Research in Computer Science and Management Studies, Volume 2, Issue (4), 2014, 15-23.

[19] Y.Bhardwaj and M.Kaushik, "A Review Paper on Virtualization and Security in Cloud Computing",
International Journal of Advanced Research in Computer Science and Software Engineering, Volume 4, Issue (3), 2014, 324-327.

[20] F.Lombardi and R.D.Pietro, "Secure virtualization for Cloud Computing", Journal of Network and Computer Applications, ELSEVIER, 2010,1-10.

[21] K.Gupta and V.K.Katiyar," "Survey of Adaptive and Dynamic Management of Cloud Datacenters", International Journal of Engineering Research and Applications,ISSN 2248-9622, 2014, 35-40.

[22] A.Uchechukwu, K.Li and Y.Shen, "Energy Consumption in Cloud Computing Data Centers", International Journal of Cloud Computing and Services Science, Volume 3, Issue(3), June 2014, 145-162.

[23] A.Banerjee, P.Agrawal and N.Ch.S.N.Iyengar, "Energy Efficiency Model for Cloud Computing", International Journal of Energy, Information and Communications, Volume 4, Issue (6) ,2013, 29-42.

[24] N.Tziritas,C.Z.Xu, T.Loukopoulos, S.U.Khan and Z.Yu, "Application-aware Workload Consolidation to Minimize both Energy Consumption and Network Load in Cloud Environments", Chinese Academy of Sciences, 2012. 